\title{
Risk assessment methodology for surface fungal infection in gymnasium workers in Lisbon: a proposal
}

\author{
C. Viegas ${ }^{1}$, C. Pinheiro ${ }^{2}$, E. Carolino ${ }^{1}$, L. Rosado ${ }^{2}$ \\ \& C. Silva Santos ${ }^{3}$ \\ ${ }^{1}$ Higher School of Health Technologies of Lisbon, \\ Polytechnic Institute of Lisbon, Portugal \\ ${ }^{2}$ National Institute of Health Dr. Ricardo Jorge, \\ Mycology Laboratory, Portugal \\ ${ }^{3}$ School of Public Health, New University of Lisbon, Portugal
}

\begin{abstract}
A descriptive study was developed to create and implement a risk assessment methodology for fungal infection in gymnasium workers. The infection was considered to occur through skin-surface contact. A pool of 10 gyms with swimming pools (the busiest of the 30 existing in Lisbon) was assessed for surface fungal contamination. Surface sampling was done before and after cleaning and disinfection procedures in 60 locations, which accounted for a total of 120 samples ( 6 from each of 10 gymnasiums at the following sites: floor surrounding the swimming pool; floor surrounding the Jacuzzi; stairs accessing the swimming pool area; floor of the training studios where most of the bare foot activities took place and changing/locker rooms for each gender. Risk assessment methodology was applied: the authors considered Severity to be dependent on the fungal species present; Probability was considered as the product between Frequency and Exposure. For each establishment, the fungal contamination mean value was calculated prior and after cleaning and disinfection in order to establish Frequency levels. Regarding Exposure, weekly hours spent in professional activity were divided into group intervals. Surprisingly, when comparing before and after cleaning and disinfection, the number of places considered to have a High Risk for fungal infection was higher in the last case. There were no training studios with High Risk of fungal
\end{abstract}


infection, and the places with the more High Risk score for fungal infection were the floors surrounding the swimming pool and the Jacuzzi.

Keywords: risk assessment, fungal infection, gymnasiums, workers.

\section{Introduction}

According to the Achilles Project, developed in 16 European countries in 2003, $34,9 \%$ of 70497 subjects suffered from feet fungal infections, Tinea pedis and onychomycosis together being the most common [1, 2]. Tinea pedis, commonly known as "athlete's foot", is an inflammatory condition and the most common form of dermatomycosis. It can be transmitted either by direct contact between two individuals or, indirectly, through contaminated objects or surfaces. Onychomycosis is usually associated with Tinea pedis and may occur due to trauma in nails during physical activity. Both diseases can occur due to exposure to contaminated surfaces with bare feet [3].

There is a wide diversity of clinical forms from Tinea pedis and onychomycosis and their etiologic agents may be dermatophytes, non dermatophyte moulds (NDM) and yeasts. Most authors diagnose as the most frequent etiological agents the dermatophytes (80 to $90 \%$ ), followed by yeasts ( 5 to $17 \%$ ) and finally NDM (2 to $12 \%$ ) [4].

Gymnasiums workers have a higher prevalence of fungal injuries, such as Tinea pedis and onychomycosis than other professional groups. This is due to their work's intrinsic characteristics, since they are more exposed to the surface's fungal contamination. This occurs not only because they attend sites most likely to be contaminated, such as showers, changing rooms and pool surrounding areas, but also because some of the activities are done with bare feet. Furthermore, synthetic clothing and occluded footwear, which retain the excessive sweating, are promoters of fungal development $[5,6]$.

Despite the possibility of adverse health effects due to fungal exposure, no health-based exposure limits have yet been proposed. This is due, in part, to the difficulty of accurately characterizing cumulative fungal spore concentrations [7] and also because, until now, epidemiological studies have failed to establish a causal relationship between the extent of fungal presence, the exposure time and specific effects on health or frequency and severity of symptoms reported [8].

In an attempt to overcome the absence of guidelines concerning surfaces fungal infection, this investigation was designed to create and implement a risk assessment methodology for fungal infection via skin-surface contact, in gymnasiums workers.

\section{Materials and methods}

A descriptive study was developed to create and implement a risk assessment methodology for fungal infection via skin-surface contact in gymnasiums workers. A pool of 10 gyms with swimming pools (the busiest of the 30 existing in Lisbon) were assessed for surface fungal contamination. 
Table 1: $\quad$ Severity levels.

\begin{tabular}{|c|c|}
\hline Severity levels & Isolated Fungi \\
\hline Null $=\mathbf{0}$ & Negative \\
\hline Moderate $=\mathbf{1}$ & NDM and yeasts \\
\hline Considerable $=\mathbf{2}$ & Pathogenic fungi (dermatophytes) \\
\hline
\end{tabular}

Surface sampling was done before and after cleaning and disinfection procedures, in 60 locations, totalizing 120 samples (6 from each of 10 gymnasiums at the following sites: floor surrounding the swimming pool; floor surrounding the Jacuzzi; stairs access to swimming pool area; floor of the training studios where most of the bare feet activity is done and changing/locker rooms from each gender).

Swabs were performed according to the International Standard ISO 18593 2004 , using a $10 \mathrm{~cm}$ square of metal disinfected with $70 \%$ alcohol solution between samples. Swabs were inoculated in triplicate on malt extract agar with chloramphenicol as a bacteria growth inhibitor (MEA) and in mycobiotic agar with cycloheximide (MA). Subsequently, these were incubated at $27^{\circ} \mathrm{C}$ (MEA for 5 to 7 days and MA for 15 to 20 days).

After laboratory processing and incubation of the collected samples, quantitative (colony forming units per square meter $-\mathrm{CFU} / \mathrm{m}^{2}$ ) and qualitative results were obtained, with the identification of the isolated fungal species. Whenever possible, filamentous fungi were identified to the species level, since adverse health effects vary according to fungal species $[10,11]$. Identification of filamentous fungi was carried out on material mounted in lactophenol blue and achieved through morphological characteristics listed in illustrated literature [11]. Yeasts were identified through biochemical API test [12].

Risk assessment methodology was applied and for the Severity criterion it was considered that infection severity and, thus, the possible injury, are closely related to the fungal species implicated (Table 1).

\section{Probability $=$ Frequency $x$ Exposure}

Probability was considered as the product between Frequency and Exposure taking into account that both Frequency and Exposure to a particular risk factor influence the probability of the outcome to occur. To establish Frequency levels, fungal contamination mean value was calculated for each establishment prior and after cleaning and disinfection.

Comparing both outcomes, the most demanding contamination values lowest mean value - were found prior to cleaning and disinfection and so, these were the values chosen for the establishment of Frequency levels. Under the Occupational Health criterion one should considered the most demanding levels to ensure protection for the most susceptible [13].

Given that the mean minimum value was 2,6 CFU $/ \mathrm{m}^{2}$ (approximately 3 $\mathrm{CFU} / \mathrm{m}^{2}$ ) and the mean value obtained from the results mean was $26,77 \mathrm{CFU} / \mathrm{m}^{2}$ (approximately $27 \mathrm{CFU} / \mathrm{m}^{2}$ ) the ranges frequency set were presented in Table 2 . 
Table 2: $\quad$ Frequency levels.

\begin{tabular}{|c|c|}
\hline Frequency levels & $\mathrm{CFU} / \mathrm{m}^{2}$ \\
\hline Minimum $=\mathbf{1}$ & $\leq \mathbf{3}$ \\
\hline Medium $=\mathbf{2}$ & $\mathbf{3}>\mathbf{X} \geq \mathbf{2 7}$ \\
\hline High $=\mathbf{3}$ & $>\mathbf{2 7}$ \\
\hline
\end{tabular}

Table 3: $\quad$ Exposure levels.

\begin{tabular}{|c|c|}
\hline Exposure levels & Weekly hours \\
\hline Minimum $=\mathbf{1}$ & $<\mathbf{1 5}$ \\
\hline Medium $=\mathbf{2}$ & {$[\mathbf{1 5}$ a 30[ } \\
\hline High $=\mathbf{3}$ & $\geq \mathbf{3 0}$ \\
\hline
\end{tabular}

Table 4: $\quad$ Risk levels.

\begin{tabular}{|c|c|}
\hline Risk levels & Obtained results \\
\hline Minimum & $\leq 3$ \\
\hline Medium & $3>X>9$ \\
\hline High & $\geq 9$ \\
\hline
\end{tabular}

Three levels of exposure were considered when analysing the time workers spent weekly in the gym (Table 3 ).

\section{Results}

According to our results, and as shown in Table 4, three risk levels were set. Regarding exposure levels, calculations were made considering only the high exposure level ( $>=30$ hours a week), because this range was the one selected by most workers.

From the 120 swabs results, 65 sites were classified as Low Risk (54,2\%), 23 sites as Medium Risk (19,2\%) and 32 sites as High Risk (26,6\%). Regarding risk levels distribution, there were more places with fungal infection High Risk classification after cleaning and disinfection (ACD) than before (BCD) (Figure 1).

Given the fact that Exposure is a constant $(E=3)$ the probability values can be totally attributed to the place itself and its conditions.

Among the facilities analysed no training studios were included in the high risk group. The area surrounding the swimming pool and Jacuzzi were the ones with the stronger association with a higher fungal infection (Figure 2).

\section{Discussion}

Regarding Severity, Faure et al. [14] also considered that the health problem is closely related to the fungal species involved. Thus, although Faure et al. 


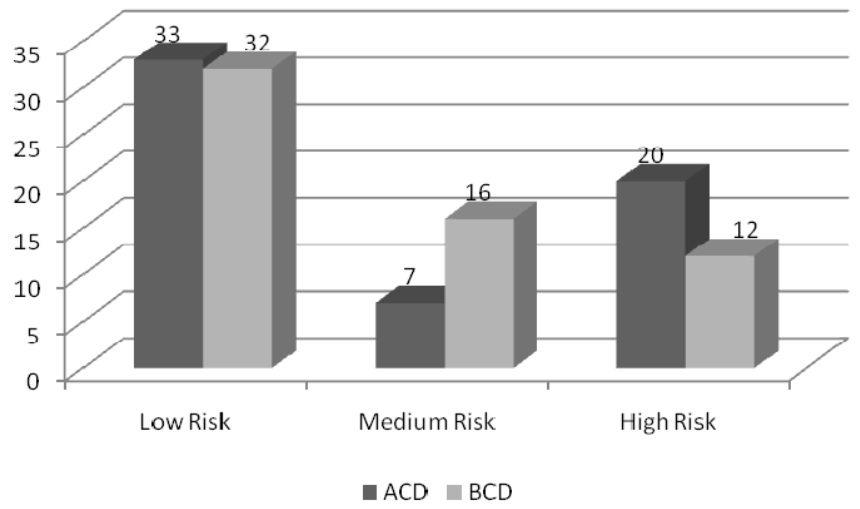

Figure 1: Risk level distributions before and after cleaning and disinfection.

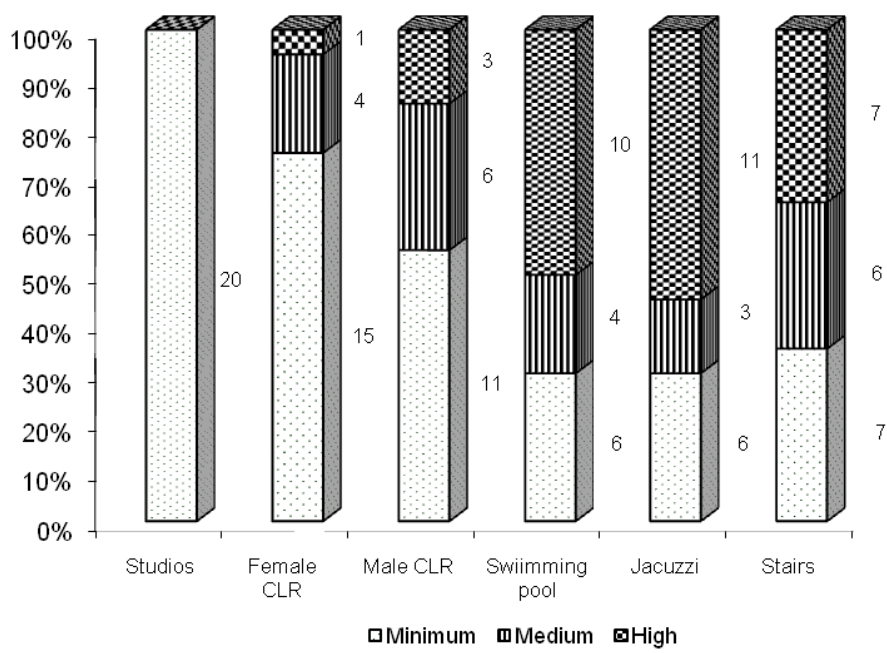

Figure 2: $\quad$ Risk level distributions in the different sites.

considered only one species - Aspergillus fumigatus - in this study Tinea pedis and onychomycosis etiologic agents, including dermatophytes, yeasts and NDM $[15,16]$ were considered.

The need to establish frequency levels arose from the wide distribution of fungal contamination means value prior and after cleaning and disinfection. Faure et al. consider $1 \mathrm{CFU} / \mathrm{m}^{3}$ because in his study $86,2 \%$ matched this value. This was not our case.

The total number of locations included in the higher risk of fungal infection group was higher after cleaning and disinfection. This situation may be due to inappropriate cleaning procedures, inadequate products and because crosscontamination can occur due to the use of common cleaning materials [17]. 
According to the results obtained from the questionnaire filled by the workers about individual and professionals variables [18], the floor surrounding the swimming pool is the second location where walking with bare feet is done more often (just after changing/locker rooms). Thus, the area more frequently included in the higher risk group was the one surrounding the swimming pool, corroborating workers increased exposure to surfaces fungal contamination. This same exposure is probably underestimated, since 114 of the 124 workers develop other physical activities different from the ones developed in a professional context [18], thus contributing to a greater risk exposure, because physical activity is associated with a high prevalence of Tinea pedis and onychomycosis [19]. Also worth mentioning is the fact that significant associations $(p<0,05)$ were found between visible injury (Tinea pedis and onychomycosis) and the number of weekly hours spent in professional activity, and between visible injury and occupation time [18].

Individual susceptibility is not considered in the proposed risk assessment methodology, and the authors acknowledge this limitation, since in fungal exposure it has been demonstrated that genetic factors play an important role in susceptibility to onychomycosis [19]. Individual susceptibility is an aspect that must be considered in risk assessment process but until now, there still this limitation in all methods that do not intend to conduct a risk assessment for a specific individual.

In terms of Severity classification, the worst case scenario was consider when dermatophytes were present in the surfaces, since most authors consider these as the most common etiologic agents of Tinea pedis and onychomycosis $[15,16]$. This does not mean, however, that other fungi (yeasts and NDM) do not also cause the mentioned diseases in workers with the same frequency and exposure with similar severity outcomes. Despite these two limitations from the proposed risk assessment methodology, it was possible to estimate fungal infection risk in the different sites, and overcome the absence of guidelines regarding surfaces fungal contamination, enabling a more effective intervention in environmental monitoring.

The obtained results with the proposed risk assessment methodology implementation can also justify the high prevalence of visible injury (Tinea pedis $18,5 \%$ and onychomycosis $19,4 \%$, a total $46,8 \%$ ) in workers from the 10 establishments studied [17], when compared to other studies, particularly in the studies conducted by Heikkila and Stubb [20] which showed a 13,0\% of onychomycosis prevalence in men, $4,3 \%$ for women and $8,4 \%$ in general population including children; Gupta et al. [21] reporting an onychomycosis prevalence of 9,1\%; Abeck et al. [22], who found 12,4\% for onychomycosis prevalence; Bramono [23] with a 3,8\% prevalence for onychomycosis ; Cheng and Chong [25] which found a 7,9\% rate; Murray and Dawber [24] reported 5\% for onychomycosis prevalence in world's population; Hamnerius et al. [26] showed a 7,8\% Tinea pedis prevalence and 2,4\% for onychomycosis; and Handog and Dayrit [27] reported 16,4\% for Tinea pedis prevalence. The difference between Tinea pedis and onychomycosis prevalence in gymnasiums workers in relation to other groups confirmed the existence of a 
serious Occupational Health problem in the studied professional group. In addition, in other studies also involving sports professionals, a similar prevalence to the one found in this study was also found: in swimmers (Tinea pedis prevalence from 15 to $20 \%$ ), runners (22\% for Tinea pedis prevalence) [28] and sports professionals who attend showers and changing rooms $(20 \%$ for onychomycosis prevalence) [29].

\section{Conclusions}

It was possible to implement a risk assessment methodology for surfaces fungal infection in gymnasiums workers, and also to estimate fungal infection risk in the different sites and overcome the absence of guidelines regarding surfaces fungal contamination, enabling a more effective intervention in environmental monitoring. Furthermore, the difference between Tinea pedis and onychomycosis prevalence in gymnasiums workers in relation to other groups confirmed the existence of a serious Occupational Health problem in the studied professional group.

\section{References}

[1] Burzykowski T, Molenberghs G, Abeck D, Haneke E, et al., High prevalence of foot diseases in Europe: results of the Achilles Project. Mycoses, 46, pp. 496 - 505, 2003.

[2] Ghannoum M, Hajeh R, Scher R, Konnikov N, et al., A large-scale North American study of fungal isolates from nails: The frequency of onychomycosis, fungal distribution and antifungal susceptibility patterns. $J$. Am. Acad. Dermatol, 43, pp. 641 - 648, 2000.

[3] Caputo R, De Boville K, Del Rosso J \& Nowicki R., Prevalence of superficial fungal infections among sports-active individuals: results from the Achilles Survey, a review of the literature. European Academy of Dermatology and Venereology, 15, pp. 312-216, 2001.

[4] Gianni C, Cerri A \& Crosti C., Non-dermatophytic onychomycosis. An underestimated entity? A study of 51 cases. Mycoses, 43, pp. 29 - 33, 2000.

[5] Attye A, Auger P, Joly J: Incidence of occult athlete's foot in swimmers. European Journal of Epidemiology. 1990; 244 - 247.

[6] Ellis D, Watson A, Marley J, Williams T: Non-dermatophytes in onychomycosis of the toenails. Br. J. Dermatol. 1997; 136: $490-493$.

[7] Bartlett K, Kennedy S, Brauer M, Van Netten C \&Dill B., Evaluation and predictive model of airborne fungal concentrations in school classrooms. Ann Occup Hyg, 48, pp., 547 - 554, 2004.

[8] Goyer N, Lavoie J, Lazure L \& Marchand G., Bioaerosols in the Workplace: Evaluation, Control and Prevention Guide. Institut de Recherche en Santé et en Sécurité du Travail du Québec, 2001.

[9] Rao C, Burge H \& Chang J., Review of quantitative standards and guidelines for fungi in indoor air. J Air Waste Manage Assoc., 46, pp. 899 908, 1996. 
[10] Hoog C, Guarro J, Gené G \& Figueiras M., (2 $2^{\text {th }}$ ed). Atlas of Clinical Fungi. Centraalbureau voor Schimmelcultures, 2000.

[11] Ghannoum M, Hajeh R, Scher R, Konnikov N, et al., A large-scale North American study of fungal isolates from nails: The frequency of onychomycosis, fungal distribution and antifungal susceptibility patterns. $J$. Am. Acad. Dermatol, 43, pp. 641 - 648, 2000.

[12] American Conference of Governmental Industrial Hygienists (ACGIH): Bioaersols: Assessment and control, Macher J (ed.). Cincinnati, Ohio: ACGIH, 1999.

[13] Faure O, Fricker-Hidalgo H, Lebeau B, Mallaret M, et al., Eight-year surveillance of environmental fungal contamination in hospital operating rooms and haematological units. Journal of Hospital Infection, 50, pp. 155 $-160,2002$.

[14] Kenna M \& Elewski B., A US epidemiological survey of superficial fungal diseases. J. Am. Acad Dermatol, 35, pp. 539 - 542, 1996.

[15] Haneke E., Fungal infections in the nail. Semin. Dermatol, 10 pp. $41-53$, 1991.

[16] Viegas C, Alves C, Carolino E, Rosado L \& Silva Santos C., Occupational exposure to fungi in gymnasiums with swimming pool in Environmental Health Risk V. WIT Transactions on Biomedicine and Health, 2009.

[17] Viegas C, Alves C, Carolino E, Rosado L \& Silva Santos C., Occupational fungal infection. Proceedings Colóquio Internacional de Segurança e Higiene Ocupacionais, 2010.

[18] Sigurgeirsson B \& Steingrimsson O., Risk Factors Associated with Onychomycosis. European Academy of Dermatology and Venereology, 18, pp. 48-51, 2004.

[19] Kamihama T, Kimura T, Hosokawa J, Ueji M, et al., Tinea pedis outbreak in swimming pools in Japan. Public Health, 111, pp. 249 - 253, 1997.

[20] Heikkila H \& Stubb S: The prevalence of onychomycosis in Finland. Br. J. Dermatol, 133, pp. 699 - 703, 1995.

[21] Gupta A, Ahmad I \& Summerbell R., Onychomycosis in children: Prevalence and treatment strategies. J. Am. Acad. Dermatol, 36, pp. $395-$ 402, 1997.

[22] Abeck D, Haneke E, Nolting S, Reinel D, et al., Onychomykose. Dt Arztebl, 97, pp. A1984 - 6, 2000.

[23] Bramono K., The Asian Achilles Survey. $6^{\text {th }}$ Asian Dermatological Congress: Bangkok; November, 2001.

[24] Murray S \& Dawber R., Onychomycosis of toenails: orthopaedic and podiatric considerations. Australas J Dermatol, 43, pp. 105 - 12, 2002.

[25] Cheng S \& Chong L., A prospective epidemiological study on Tinea pedis and onychomycosis in Hong Kong. CMJ, 115 (6), pp. $860-865,2002$.

[26] Hamnerius N, Berglund J \& Faergemann J., Clinical and laboratory investigations. Pedal dermatophyte infection in psoriasis. British Journal of Dermatology, 150, pp. 1125 - 1128, 2004.

[27] Handog E \& Dayrit J: Mycology in the Philippines, Revisited. Jpn. J. Med. Mycol, 46, pp. $71-76,2005$. 
[28] Gudnadóttir G, Hilmarsdóttir I \& Sigurgeirsson B., Onychomycosis in Iceland swimmers. Acta Derm Venereol, 79, pp. 376-377, 1999.

[29] Ellis D, Watson A, Marley J \& Williams T., Non-dermatophytes in onychomycosis of the toenails. Br. J. Dermatol, 136, pp. 490 - 493, 1997. 